\title{
Impedance Operator Description of a Metasurface with Electric and Magnetic Dipoles
}

\author{
Didier Felbacq \\ Université de Montpellier, Laboratoire Charles Coulomb UMR CNRS-UM 5221, 34095 Montpellier, France \\ Correspondence should be addressed to Didier Felbacq; didier.felbacq@umontpellier.fr
}

Received 5 August 2015; Accepted 4 November 2015

Academic Editor: Ivan D. Rukhlenko

Copyright (C) 2015 Didier Felbacq. This is an open access article distributed under the Creative Commons Attribution License, which permits unrestricted use, distribution, and reproduction in any medium, provided the original work is properly cited.

\begin{abstract}
A metasurface made of a collection of nanoresonators characterized by an electric dipole and a magnetic dipole was studied in the regime where the wavelength is large with respect to the size of the resonators. An effective description in terms of an impedance operator was derived.
\end{abstract}

\section{Introduction}

Metasurfaces are the bidimensional analogue of metamaterials [1]. They are made of basic cells containing resonant elements disposed periodically or not on a surface. The cells can contain one or several elements (nanoparticles, nanoantennas, etc.) whose aim is to produce a dephasing of some incident field, whose wavelength is large with respect to the size of the cells. The collection of cells is then able to produce a collective effect resulting in refracting and transmitting properties far from that of a homogeneous material [2]. For instance, it was shown in [3] that they could be used to design flat achromatic lenses. Metasurfaces are thus a wide generalization of subwavelength diffraction gratings, where the basic cell can be quite complicated. The periodic structure of gratings can be relaxed so as to obtain interesting effect on the polarization of the incident field [4]. In this context, we study the field diffracted by a periodic set of linear nanoresonators, electromagnetically characterized by their scattering matrix $S$. We are interested in the regime where the wavelength is much larger than the size of the nanoresonators. We proceed to an asymptotic analysis related to homogenization theory $[5,6]$. The field diffracted by the structure is derived and it is shown that it is characterized by an impedance operator. Our results extend to electric and magnetic dipoles results in [7] where only electric dipoles were considered.

\section{Setting of the Problem}

The structure under study is made of an infinite number of resonators invariant along $z$, periodically disposed at points $M_{p}=(p \times d, 0)$, where $d$ is the period and $p \in \mathbf{Z}$. Each scatterer at position $M_{p}$ is characterized in the frequency domain by a scattering matrix $S(\omega)$ as well as by electromagnetic parameters $\gamma_{s}$ and $\delta_{s}$. We assume that the wavelength in vacuum $\lambda=2 \pi c / \omega$ is much larger than the size of the resonators, which are assumed to be contained in a cylinder of diameter $a$. We therefore consider a linearly polarized incident field $U^{i} e^{-i \omega t} e_{z}$, where $U^{i}(x, y)=e^{i(\alpha x \pm \beta y)}$ and $\alpha=k_{0} \sin \theta, \theta$ is an angle of incidence, and $k_{0}=\omega / c$. According to the polarization $\gamma_{s}$ and $\delta_{s}$ can be either the relative permittivity or relative permeability.

The stationary scattering problem considered therefore reads the following: find a field $U \in H_{\text {loc }}^{1}\left(\mathbb{R}^{2}\right)$ (i.e., the space of locally square integrable functions with a locally square integrable weak derivative) satisfying $\operatorname{div}(\gamma \nabla U)+k_{0}^{2} \delta U=0$ in the sense of Schwartz distribution in $\mathbb{R}^{2}$ and such that $U^{s}=U-U^{i}$ satisfies the outgoing wave conditions:

(i) For $y>0$ : $\operatorname{Im}\left(U^{s} \nabla U^{s}\right)>0$.

(ii) For $y<0$ : $\operatorname{Im}\left(U^{s} \nabla U^{s}\right)<0$. 
The functions $\gamma$ and $\delta$ are equal, respectively, to $\gamma_{s}$ and $\delta_{s}$ inside the scatterers and to 1 outside. The following holds; see [8].

Proposition 1. Apart possibly from a discrete set of wavenumbers $k_{1}, k_{2}, \ldots$, the field $U$ exists and is unique.

Our point is to provide a simplified expression of the scattering problem by means of an impedance operator.

Let $T_{d}$ denote the translation along $x$ of amplitude $d$; that is, $T_{d}(f)(x)=f(x-d)$. For later purpose, we define $Y^{*}=$ ] $-\pi / d, \pi / d], K=2 \pi / d, \alpha_{n}=\alpha+n K$, and $\beta_{n}=\sqrt{k_{0}^{2}-\alpha_{n}^{2}}$, for $n \in \mathbb{Z}$. For $\alpha_{n}^{2}>k_{0}^{2}$, we impose $i \beta_{n}<0$. In the following, we denote $\mathbb{T}=\mathbb{R} / d \mathbb{Z}$.

Let $H=-\operatorname{div}(\gamma \nabla \cdot)-k_{0}^{2} \delta$ and let us define for $\alpha \in Y^{*}$ the field of Hilbert spaces $L_{\alpha}^{2}(\mathbb{T})=\left\{u ; u e^{-i \alpha x} \in L^{2}(\mathbb{T})\right\}$. It obviously holds the following.

Lemma 2. The commutator of $T_{d}$ and $H$ vanishes: $\left[T_{d}, H\right]=$ 0 .

Applying Floquet-Bloch analysis [9], we obtain the following.

Proposition 3. The operator $H$ has a direct integral decomposition $H=\int_{Y^{*}}^{\oplus} H_{\alpha}(d \alpha / K)$ where $H_{\alpha}=-\operatorname{div}(\gamma \nabla \cdot)-k_{0}^{2} \delta$ with domain $D\left(H_{\alpha}\right)=\left\{L_{\text {loc }}^{2}\left(\mathbb{R}_{y} ; L_{\alpha}^{2}(\mathbb{\mathbb { T }})\right) \cap H_{\text {loc }}^{2}(Y \times \mathbb{R})\right\}$.

\section{Multiple Scattering Approach}

The incident field has the expansion [10] $U^{i}(x, y)=$ $\sum_{n} a_{n} J_{n}\left(k_{0} r\right) e^{i n \theta}$. For one scatterer alone, the incident field gives rise to a field $U_{p}^{s}(x, y)=\sum_{n} s_{n}^{p} \varphi_{n}(x, y)$ where $\varphi_{n}(x, y)=$ $H_{n}^{(1)}\left(k_{0} r\right) e^{i n \theta} . J_{n}$ (resp. $\left.H_{n}^{(1)}\right)$ is a Bessel (resp., Hankel) function. For the infinite set of scatterers, this gives a diffracted field that reads

$$
U^{s}(x, y)=\sum_{p, n} s_{n}^{p} \varphi_{n}(x-p d, y)
$$

Multiple scattering theory [10] allows to write that for $p=0$

$$
\widehat{b^{0}}=(1-S \Sigma)^{-1} S \widehat{a}
$$

where $\widehat{b^{0}}=\left(\ldots, b_{-n}^{0}, \ldots, b_{n}^{0}, \ldots\right)^{T}$ and $\widehat{a}=\left(\ldots, a_{-n}, \ldots\right.$, $\left.a_{n}, \ldots\right)^{T}$. The matrix $\Sigma$ is given by

$$
\Sigma\left(k_{0}, \alpha_{0}\right)=\sum_{m \neq 0} e^{i \alpha_{0} m} T_{0 m}
$$

Here Proposition 3 was used through the introduction of a Bloch phase $e^{i \alpha_{0} m}$. In this expression $\left(T_{0 m}\right)_{p q}=$ $e^{i(p-q) \theta_{0}^{m}} H_{p-q}^{(1)}\left(k_{0}|m| d\right)$; that is,

$$
T_{0 m}=\left(\begin{array}{ccccc}
\ddots & \vdots & \vdots & \vdots & \cdots \\
\cdots & H_{0}\left(k_{0}|m| d\right) & -\epsilon_{m} H_{1}\left(k_{0}|m| d\right) & H_{2}\left(k_{0}|m| d\right) & \cdots \\
\cdots & \epsilon_{m} H_{1}\left(k_{0}|m| d\right) & H_{0}\left(k_{0}|m| d\right) & -\epsilon_{m} H_{1}\left(k_{0}|m| d\right) & \cdots \\
\cdots & H_{2}\left(k_{0}|m| d\right) & \epsilon_{m} H_{1}\left(k_{0}|m| d\right) & H_{0}\left(k_{0}|m| d\right) & \cdots \\
\cdots & \vdots & \vdots & \vdots & \ddots
\end{array}\right),
$$

where $\epsilon_{m}=\operatorname{sign}(m)$ (note that $\left.e^{i \theta_{0}^{m}}=-\operatorname{sign}(m)\right)$. The following series [11] indexed by $p$ appear:

$$
\Sigma_{p}=\sum_{m \neq 0} e^{i m \alpha_{0}} \epsilon_{m}^{p} H_{p}\left(k_{0}|m| d\right) .
$$

And the entries of the matrix $\Sigma\left(k_{0}, \alpha_{0}\right)$ are $\left(\Sigma\left(k_{0}, \alpha_{0}\right)\right)_{p q}=$ $\Sigma_{p-q}$.

In the regime where $k_{0} a \ll 1$, the cylinder can be described by a $3 \times 3$ scattering matrix (this corresponds to an electric dipole and a magnetic dipole) and the field by 3 coefficients $b_{-1}, b_{0}$, and $b_{1}[10]$. Therefore, only 3 series are involved: $\Sigma_{0}, \Sigma_{1}$, and $\Sigma_{2}$. It holds that

$$
\Sigma\left(k_{0}, \alpha_{0}\right)=\left(\begin{array}{ccc}
\Sigma_{0} & -\Sigma_{1} & \Sigma_{2} \\
\Sigma_{1} & \Sigma_{0} & -\Sigma_{1} \\
\Sigma_{2} & \Sigma_{1} & \Sigma_{0}
\end{array}\right) .
$$

In the extreme limit $(a \ll d)$ where the scatterers are very small as compared to the wavelength and the period, the scattering matrix $S(\omega)$ reduces to a scalar matrix $s_{0}(\omega)$ : the scatterers are thus dipoles with a dipole moment along $e_{z}$ and the only involved series is $\Sigma_{0}$; this situation was addressed in [7]. The multiple scattering relation (2) then becomes

$$
b_{0}^{0}\left(k_{0}, \alpha_{0}\right)=\left(1-S_{0} \Sigma_{0}\right)^{-1} S_{0}
$$

where the series $\Sigma_{0}$ can be written $[7,12,13]$ :

$$
\Sigma_{0}\left(k_{0}, \alpha_{0}\right)=\sum_{m \neq 0} e^{i k m d} H_{0}\left(k_{0}|m| d\right) .
$$

For the more general case of an electric dipole and a magnetic dipole the following asymptotic expressions hold in the limit $k_{0} d \ll 1[14]$. 
Proposition 4. Consider the following:

$$
\begin{aligned}
& \Sigma_{0}\left(k_{0}, \alpha_{0}\right) \sim-1-\frac{2 i}{\pi} \gamma+\frac{2 i}{\pi} \ln \left(\frac{2 K}{k_{0}}\right)+\frac{K}{\pi \beta_{0}}, \\
& \Sigma_{1}\left(k_{0}, \alpha_{0}\right) \sim \frac{\alpha_{0}}{\pi k_{0}}\left(-2+i \frac{K}{\beta_{0}}\right), \\
& \Sigma_{2}\left(k_{0}, \alpha_{0}\right) \sim \frac{K}{\pi k_{0}^{2}} \frac{\beta_{0}^{2}-\alpha_{0}^{2}}{\beta_{0}}-\frac{i}{\pi k_{0}^{2}}\left(\frac{K^{2}}{3}-\beta_{0}^{2}+\alpha_{0}^{2}\right) .
\end{aligned}
$$

\section{Scattering Properties of the Meta Surface}

Define $P_{z}=b_{0}^{0}$ the electric moment and $M=\left(M_{x}, M_{y}\right)=$ $\left(\left(b_{1}^{0}+b_{-1}^{0}\right), i\left(b_{1}^{0}-b_{-1}^{0}\right)\right)$ the magnetic moment. We write $\mathbf{m}=$ $M_{x}+i M_{y}, \mathbf{m}^{*}=M_{x}-i M_{y}$, and $\kappa_{n}^{+}=\left(\alpha_{n}, \beta_{n}\right), \kappa_{n}^{-}=\left(\alpha_{n},-\beta_{n}\right)$.

We can now state the following.

Theorem 5. The total field has the following expression:

$$
\begin{aligned}
& y>a: U(x, y)=e^{i\left(\alpha_{0} x-\beta_{0} y\right)}+\sum_{n} r_{n} e^{i\left(\alpha_{n} x+\beta_{n} y\right)}, \\
& y<a: U(x, y)=\sum_{n} t_{n} e^{i\left(\alpha_{n} x-\beta_{n} y\right)}
\end{aligned}
$$

where

$$
\begin{aligned}
& r_{n}=\frac{K}{\pi \beta_{n}}\left(P_{z}+i M \cdot \kappa_{n}^{+}\right), \\
& t_{n}=\delta_{n 0}+\frac{K}{\pi \beta_{n}}\left(P_{z}+i M \cdot \kappa_{n}^{-}\right) .
\end{aligned}
$$

Proof. We start with the following relation, obtained from Poisson formula:

$$
\sum_{n} H_{0}\left(k_{0}\left|r-n d e_{x}\right|\right) e^{i k n d}=\frac{2}{d} \sum_{n} \frac{1}{\beta_{n}} e^{i\left(\alpha_{n} x+\beta_{n}|y|\right)} .
$$

Upon applying the operator $\partial=\partial_{x}+i \partial_{y}$, using the fact that the series on the right-hand side is normally convergent (thanks to the term $e^{i \beta_{n}|y|}$ ) and using the relation $\partial H_{0}(r)=-H_{1}(r) e^{i \theta}$, we obtain

$$
\begin{aligned}
- & k_{0} \sum_{n} \varphi_{1}(x-n d, y) e^{i k n d} \\
= & \frac{2}{d} \sum_{n} \frac{\left(i \alpha_{n}-\beta_{n} \epsilon\right)}{\beta_{n}} e^{i\left(\alpha_{n} x+\beta_{n}|y|\right),}
\end{aligned}
$$

where $\epsilon=\operatorname{sign}(y)$. Therefore we get

$$
\begin{aligned}
& U^{s}(x, y)=\frac{2}{d} \\
& \cdot \sum_{n} \frac{b_{0}^{0}+i \alpha_{n}\left(b_{1}^{0}+b_{-1}^{0}\right)+\left(b_{-1}^{0}-b_{1}^{0}\right) \beta_{n} \epsilon}{\beta_{n}} e^{i\left(\alpha_{n} x+\beta_{n}|y|\right)} .
\end{aligned}
$$

The result follows after some simple algebra.

A simple, but interesting corollary is as follows.
Corollary 6. As $n \sim+\infty$,

$$
\begin{aligned}
& r_{n} \sim \frac{K}{\pi} \mathbf{m}=\frac{2 K b_{-1}^{0}}{\pi}, \\
& t_{n} \sim \frac{K}{\pi} \mathbf{m}^{*}=\frac{2 K b_{1}^{0}}{\pi} .
\end{aligned}
$$

We are not a priori in the homogenization regime where $k_{0} d \ll 1$ and hence there can be several reflected and transmitted orders. In expression (10), the propagative waves (i.e., the diffractive orders of the grating) correspond to the $\beta_{n}$ 's that are real. They are labelled by the finite set $U=\{n \in$ $\left.\mathbb{Z}, \beta_{n} \in \mathbb{R}^{+}\right\}$. The evanescent waves are labelled by the infinite set $U^{+}=\left\{n \in \mathbb{Z}, i \beta_{n} \in \mathbb{R}^{-}\right\}$.

\section{Impedance Operator Formulation}

Our point is now to replace the set of nanoresonators by a metasurface $\mathbb{S}$ which is simply the line $y=0$. This requires to specify the boundary conditions there in terms of an impedance operator.

So, consider the continuation of the field $U$ obtained by making $a=0$ in (10). The continued field is still denoted as $U$. It is a singular distribution. To handle this situation, let us introduce the following fields of Sobolev spaces

$$
\begin{aligned}
& H_{\alpha}^{s / 2}(Y) \\
& \quad=\left\{u=\sum_{n} u_{n} e^{i \alpha_{n} x} ; \sum_{n}\left(1+\left|k_{n}\right|^{2}\right)^{s / 2}\left|u_{n}\right|^{2}<+\infty\right\}
\end{aligned}
$$

and the dual spaces

$$
\begin{aligned}
& H_{\alpha}^{-s / 2}(Y) \\
& \quad=\left\{u=\sum_{n} u_{n} e^{i \alpha_{n} x} ; \sum_{n}\left(1+\left|k_{n}\right|^{2}\right)^{-s / 2}\left|u_{n}\right|^{2}<+\infty\right\} .
\end{aligned}
$$

Let $\mathscr{Z}$ be the pseudodifferential operator defined by $\mathscr{Z}[u]=$ $v$, where

$$
\begin{aligned}
& u(x)=\sum_{n} u_{n} e^{i \alpha_{n} x}, \\
& v(x)=\sum_{n} i \beta_{n} u_{n} e^{i \alpha_{n} x} .
\end{aligned}
$$

It is straightforward to show the following.

Proposition 7. $\mathscr{Z}$ is continuous and invertible from $H_{\alpha}^{-s / 2}(Y)$ to $H_{\alpha}^{-s / 2-1}(Y)$, for $s>1$.

The inverse of $\mathscr{Z}$ is the admittance operator $\mathcal{Y}$ defined by $v=\mathscr{Y}[u]$. 
Let us denote $F$ as an element of $H_{\alpha}^{-s / 2}(Y) \times H_{\alpha}^{-s / 2-1}(Y)$, representing the discontinuity of the field and its derivative through $\mathbb{S}$. The traces of the field and its derivative are

$$
\begin{aligned}
& F^{+}=\left(\begin{array}{c}
U\left(x, 0^{+}\right) \\
\frac{\partial U}{\partial y}\left(x, 0^{+}\right)
\end{array}\right), \\
& F^{-}=-\left(\begin{array}{c}
U\left(x, 0^{-}\right) \\
\frac{\partial U}{\partial y}\left(x, 0^{-}\right)
\end{array}\right) .
\end{aligned}
$$

By definition, it holds that $F^{+}+F^{-}=F$. The Calderòn projectors [15] $\mathscr{P}^{+}$and $\mathscr{P}^{-}$are defined by $F^{+}=\mathscr{P}^{+} F$ and $F^{-}=\mathscr{P}^{-} F$. The preceding shows the following.

Proposition 8. Consider the following:

$$
\begin{aligned}
& \mathscr{P}^{+}=\frac{1}{2}\left(\begin{array}{cc}
1 & \mathscr{Y} \\
\mathscr{X} & 1
\end{array}\right), \\
& \mathscr{P}^{-}=\frac{1}{2}\left(\begin{array}{cc}
1 & -\mathscr{Y} \\
-\mathscr{Z} & 1
\end{array}\right) .
\end{aligned}
$$

Obviously, it holds that $\mathscr{P}^{+}+\mathscr{P}^{-}=\llbracket,\left(\mathscr{P}^{+}\right)^{2}=\mathscr{P}^{+}$, $\left(\mathscr{P}^{-}\right)^{2}=\mathscr{P}^{-}$, and $\mathscr{P}^{+} \mathscr{P}^{-}=\mathscr{P}^{-} \mathscr{P}^{+}=0$, as it should.

The transmission conditions on $\mathbb{S}$ can be written as follows:

$$
\begin{aligned}
& {\left[U\left(x, 0^{+}\right)-U\left(x, 0^{-}\right)\right]=\frac{2 i K M_{y}}{\pi} \sum_{n} e^{i \alpha_{n} x}} \\
& =\frac{2 i K M_{y}}{\pi} \sum_{n} \frac{1}{i \beta_{n} r_{n}} i \beta_{n} r_{n} e^{i \alpha_{n} x}, \\
& {\left[\frac{\partial U}{\partial y}\left(x, 0^{+}\right)-\frac{\partial U}{\partial y}\left(x, 0^{-}\right)\right]} \\
& =\sum_{n} i \beta_{n}\left(\delta_{n 0}+r_{n}+t_{n}\right) e^{i \alpha_{n} x} \\
& =\sum_{n} i \beta_{n} \frac{\left(\delta_{n 0}+r_{n}+t_{n}\right)}{\delta_{n 0}+r_{n}} r_{n} e^{i \alpha_{n} x} .
\end{aligned}
$$

This suggests to define the following pseudodifferential operators, acting on $u=\sum_{n} u_{n} e^{i \alpha_{n} x}$ :

$$
\begin{aligned}
& \mathscr{X}[u](x)=\sum_{n}\left(1+\frac{t_{n}}{\delta_{n 0}+r_{n}}\right) u_{n} e^{i \alpha_{n} x}, \\
& \mathscr{W}[u](x)=\frac{2 i K M_{y}}{\pi} \sum_{n} \frac{1}{r_{n}} u_{n} e^{i \alpha_{n} x} .
\end{aligned}
$$

Both $r_{n}$ and $t_{n}$ are bounded with respect to $n$ (see (15)); hence see the following.

Proposition 9. The pseudodifferential operators $\mathscr{X}$ and $\mathscr{W}$ are isomorphism of $L_{\alpha}^{2}(\mathbb{T})$.
These conditions can be rewritten conveniently in the operator form.

Theorem 10. The traces of the field $U(x, y)$ diffracted by the metasurface under the illumination of a plane wave $U^{i}$ satisfy the impedance conditions:

$$
\mathscr{T} F^{+}=F^{-},
$$

where

$$
\mathscr{T}=\left(\begin{array}{cc}
1 & -\mathscr{Y} \mathscr{W} \\
-\mathscr{Z} \mathscr{X} & 1
\end{array}\right) .
$$

The operator $\mathscr{T}$ is the transfer matrix of the meta surface. The discontinuity of the (effective) field $U$ at $y=0$ is due to the existence of a magnetic dipole moment. In the homogenization limit of very large wavelengths, that is, larger than the wavelengths corresponding to magnetic resonances and larger than twice the period, there are only one transmitted and one reflected wave; the evanescent waves can be discarded and the magnetic resonances have no effect; we then have the following.

Proposition 11. For a wavelength $\lambda \gg 2 d$ and larger than the largest magnetic resonance, the propagative part of the field is given by

$$
\begin{aligned}
& y>0: U(x, y)=e^{i\left(\alpha_{0} x-\beta_{0} y\right)}+r_{0} e^{i\left(\alpha_{0} x+\beta_{0} y\right)}, \\
& y<0: U(x, y)=t_{0} e^{i\left(\alpha_{0} x-\beta_{0} y\right)},
\end{aligned}
$$

where $r_{0}=2 b_{0}^{0} / d \beta_{0}$ and $t_{0}=1+r_{0}$. The transfer matrix becomes

$$
\mathscr{T}=\left(\begin{array}{ccc}
1 & 0 \\
-2 i \beta_{0} \frac{r}{1+r} & 1
\end{array}\right) .
$$

The form found for the transfer matrix matches that obtained in another context in [12].

\section{Conclusion}

The scattering of a plane wave by a grating of nanorods was described in the framework of metasurfaces by exhibiting an impedance condition. This takes into account both the electric and the magnetic dipoles characterizing each nanorod. This study can be generalized to higher multipoles but also to nonperiodic, for instance, quasiperiodic, structures [16] and to elementary scatterers deposited on an arbitrary smooth surface as well. A similar approach was used in [17] to study the coupling of a quantum emitter with the modes supported by the metasurface; see also [18]. The proposed formalism can be used in this context to analyze the role of resonances.

\section{Conflict of Interests}

The author declares that there is no conflict of interests regarding the publication of this paper. 


\section{References}

[1] C. L. Holloway, E. F. Kuester, J. A. Gordon, J. O'Hara, J. Booth, and D. R. Smith, "An overview of the theory and applications of metasurfaces: the two-dimensional equivalents of metamaterials," IEEE Antennas and Propagation Magazine, vol. 54, no. 2, pp. 10-35, 2012.

[2] N. Yu and F. Capasso, "Flat optics with designer metasurfaces," Nature Materials, vol. 13, no. 2, pp. 139-150, 2014.

[3] M. Khorasaninejad, F. Aieta, P. Kanhaiya et al., "Achromatic metasurface lens at telecommunication wavelengths," Nano Letters, vol. 15, no. 8, pp. 5358-5362, 2015.

[4] X. Ma, M. Pu, X. Li et al., "A planar chiral meta-surface for optical vortex generation and focusing," Scientific Reports, vol. 5, Article ID 10365, 2015.

[5] L. Tartar, The General Theory of Homogenization, Springer, New York, NY, USA, 2009.

[6] D. Felbacq and G. Bouchitté, "Homogenization of a set of parallel fibres," Waves in Random Media, vol. 7, no. 2, pp. 245256, 1997.

[7] D. Felbacq, "Layer homogenization of a 2D periodic array of scatterers," Photonics and Nanostructures-Fundamentals and Applications, vol. 11, no. 4, pp. 436-441, 2013.

[8] A.-S. Bonnet-Bendhia and F. Starling, "Guided waves by electromagnetic gratings and non-uniqueness examples for the diffraction problem," Mathematical Methods in the Applied Sciences, vol. 17, no. 5, pp. 305-338, 1994.

[9] M. Reed and B. Simon, Methods of Modern Mathematical Physics, vol. 4 of Analysis of Operators, Academic Press, New York, NY, USA, 1978.

[10] D. Felbacq, G. Tayeb, and D. Maystre, "Scattering by a random set of parallel cylinders," Journal of the Optical Society of America A, vol. 11, no. 9, pp. 2526-2538, 1994.

[11] C. M. Linton, "Lattice sums for the Helmholtz equation," SIAM Review, vol. 52, no. 4, pp. 630-674, 2010.

[12] F. Zolla, D. Felbacq, and G. Bouchitté, "Bloch vector dependence of the plasma frequency in metallic photonic crystals," Physical Review E, vol. 74, no. 5, Article ID 056612, 2006.

[13] P. A. Belov, S. A. Tretyakov, and A. J. Viitanen, "Dispersion and reflection properties of artificial media formed by regular lattices of ideally conducting wires," Journal of Electromagnetic Waves and Applications, vol. 16, no. 8, pp. 1153-1170, 2002.

[14] A. I. Cabuz, Electromagnetic metamaterials-from photonic crystals to negative index composites [Ph.D. thesis], Université Montpellier II, 2007, https://hal.archives-ouvertes.fr/tel00161428

[15] M. Cessenat, Mathematical Methods in Electromagnetism, World Scientific, Singapore, 1996.

[16] Z. V. Vardeny, A. Nahata, and A. Agrawal, "Optics of photonic quasicrystals," Nature Photonics, vol. 7, no. 3, pp. 177-184, 2013.

[17] D. Felbacq, "Weak and strong coupling of a quantum emitter with a meta-surface," Superlattices and Microstructures, vol. 78, pp. 79-87, 2015.

[18] A. Cazé, R. Pierrat, and R. Carminati, "Strong coupling to two-dimensional Anderson localized modes," Physical Review Letters, vol. 111, no. 5, Article ID 053901, 2013. 


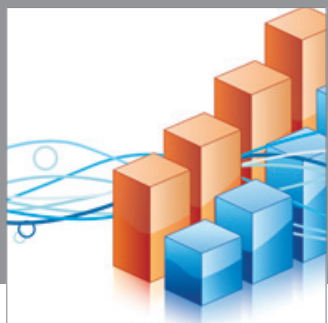

Advances in

Operations Research

mansans

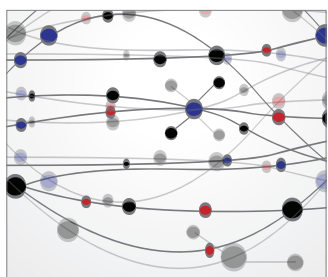

The Scientific World Journal
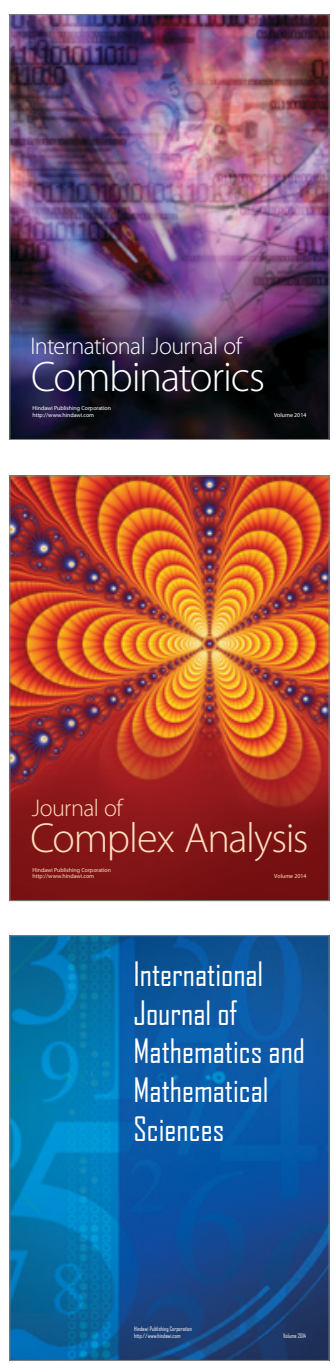
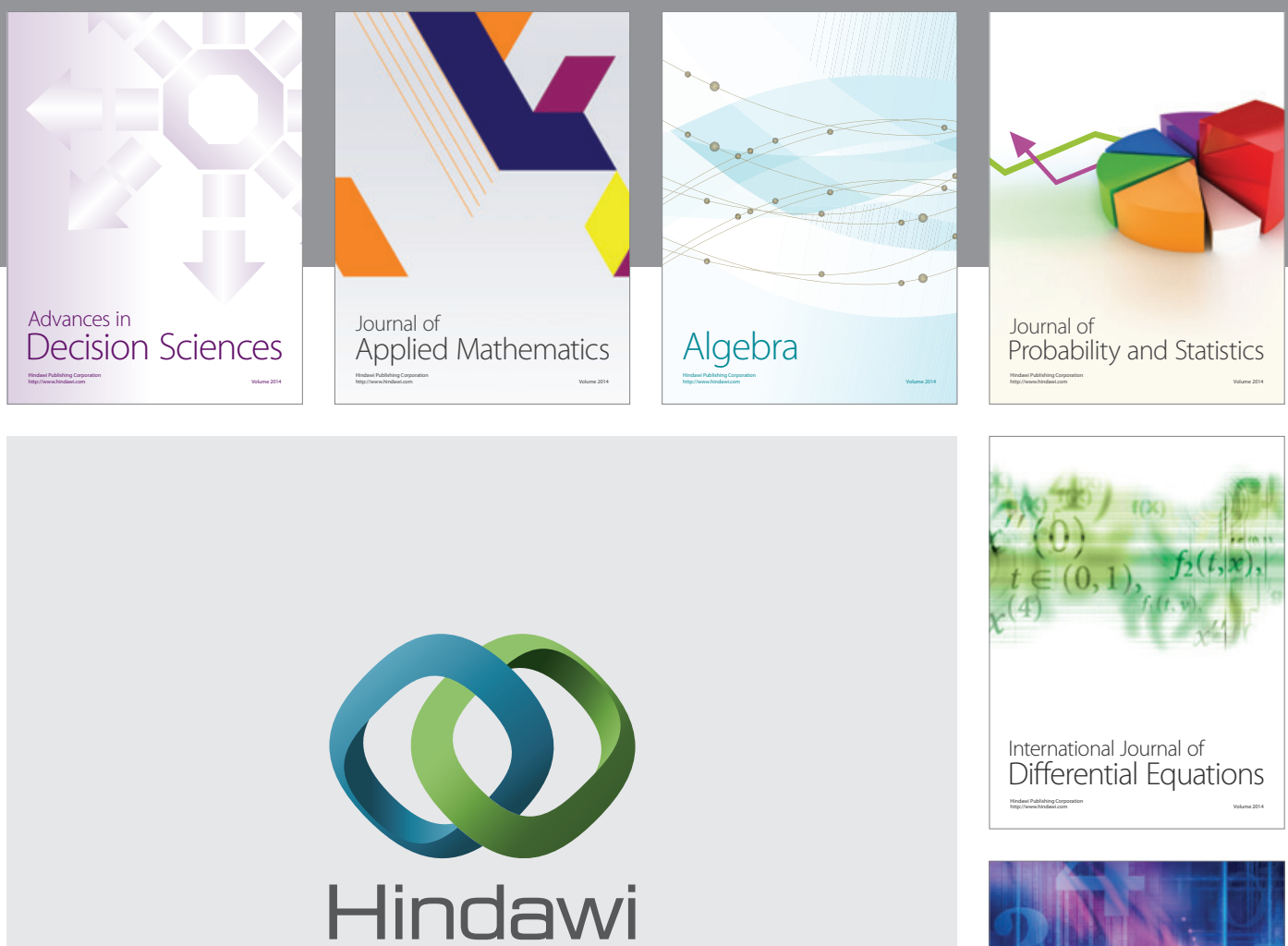

Submit your manuscripts at http://www.hindawi.com
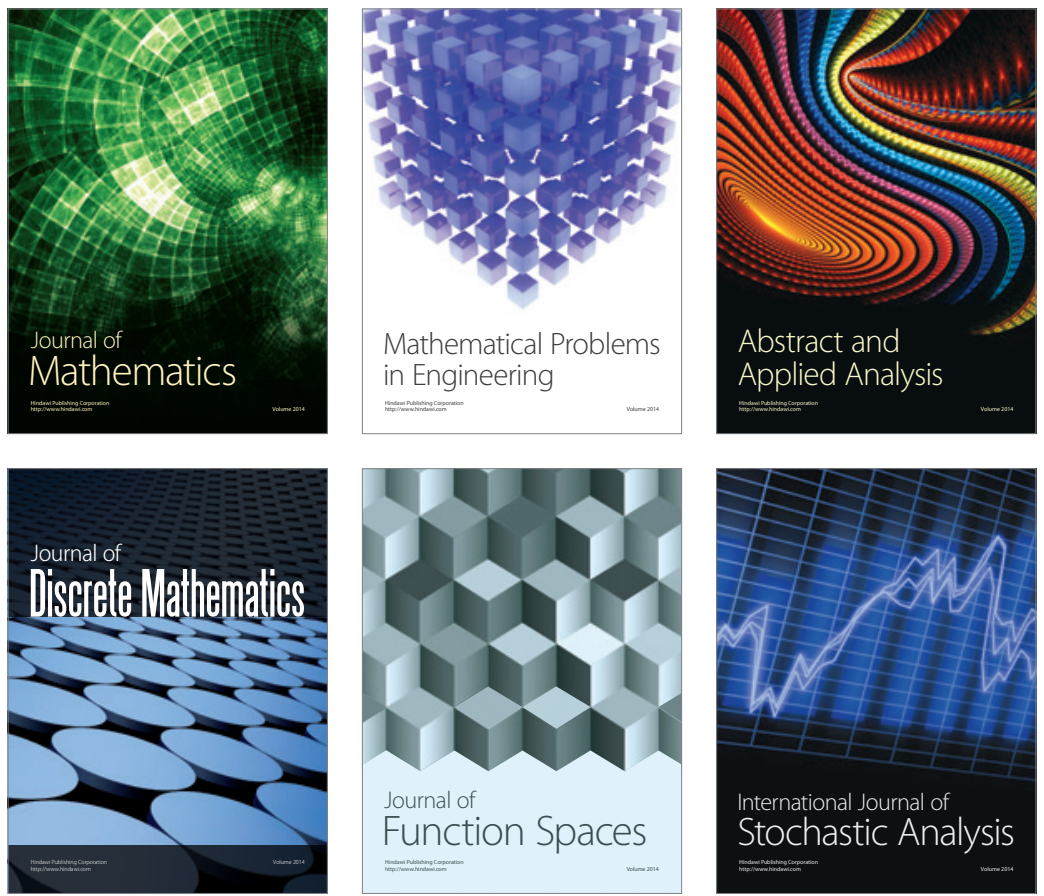

Journal of

Function Spaces

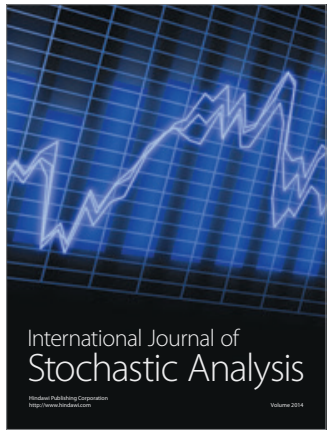

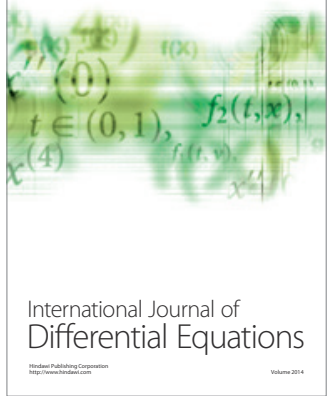
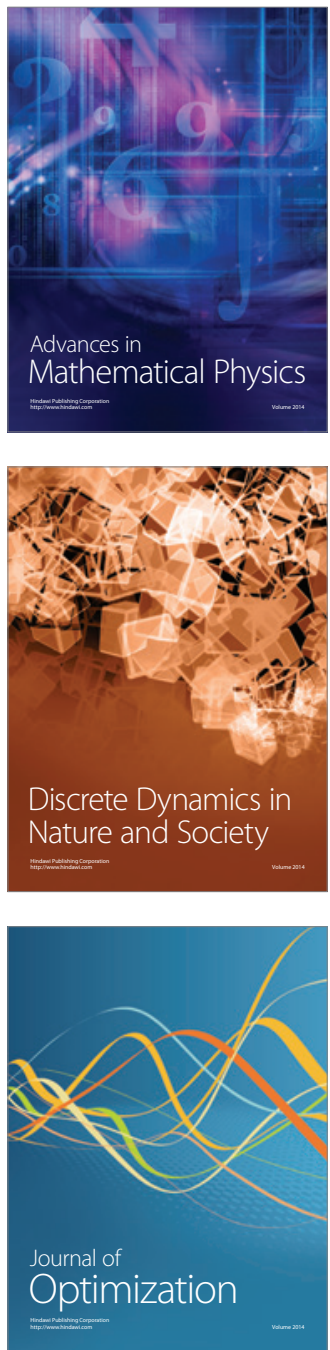